\title{
Desenvolvimento de um medidor por indução de baixo custo para controle de consumo de energia elétrica em unidades residenciais de baixa renda na Amazônia
}

\author{
Development of a low-cost induction meter for control of electricity \\ consumption in low-income housing of the Amazon:
}

Cristiano Torres do Amaral cristiano.amaral@sipam.gov.br SIPAM-GPERS/UNIR

\author{
Artur Salvador \\ artursalvador@gmail.com \\ UNIR \\ Laya Fernanda Belici \\ Siborde \\ lay.belici@hotmail.com \\ UNIR \\ Lorena Paula Mendonça \\ Guedes \\ lorenguedes@gmail.com \\ UNIR
}

\begin{abstract}
Resumo
Este artigo apresenta o desenvolvimento de um protótipo de baixo custo para mensurar corrente alternada por indução como proposta para medição de energia em unidades habitacionais de baixa renda. O sistema é de simples funcionamento e, uma vez conectado, um sensor realiza a leitura da medição de energia por comandos do telefone celular com aplicativo via bluetooth. O projeto pode ser empregado em diversos smartphones ou tablets com sistema operacional Android. O protótipo tem um display OLED para a visualização da corrente que é consumida e que circula pelo aparelho, sendo de fácil construção, e ainda tem possíveis aplicações didáticas em laboratório de instrumentação.
\end{abstract}

Palavras-chave: Android. Eficiência energética. Protótipo.

\begin{abstract}
This paper presents the development of a low cost prototype to measure alternating current through induction as a proposal for energy measurement in low income housing units. The system is simple to operate and once connected, a sensor performs the reading of the energy measurement by cellular phone commands with application via Bluetooth. The project can be used on several smartphones or tablets with Android operating system. The prototype has an OLED display for the visualization of the current that is consumed and circulates through the device, being of easy construction, and also, with possible didactic applications in instrumentation laboratory.
\end{abstract}

Keywords: Android. Energy efficiency. Prototype.

\section{Introdução}

Nos últimos anos, houve um aumento significativo das faturas de energia elétrica, bem como aplicação de bandeiras tarifárias para contenção do consumo de energia por meio do sobrefaturamento compulsório. Esses aumentos colocaram muitas famílias em dificuldades financeiras. Em alguns casos, o descontrole ocorre porque as pessoas têm dificuldade de acompanhar o consumo de energia de suas casas. A falta de conhecimento técnico, o uso de medidores de energia obsoletos ou de visualização complexa, entre outros fatores, dificultam o monitoramento do consumo, principalmente das unidades familiares de baixa renda. De acordo com dados fornecidos pelo Governo Federal relativos ao benefício Tarifa Social de Energia Elétrica, entre 2000 e 2016, houve um aumento de cerca de 38\% no consumo de energia elétrica das famílias que estavam cadastradas no programa. $\mathrm{O}$ aumento da carga tributária, descuido e a falta de controle motivaram o aumento gradativo do faturamento, o qual também estimula a revisão dos benefícios sociais e, por isso, famílias podem perder os benefícios sociais de acordo com a atualização de cadastral que considera o consumo de energia elétrica residencial (ANEEL, 2012).

O agravamento da crise econômica e a necessidade acompanhamento do consumo de energia elétrica por parte das famílias de baixa renda na Amazônia sensibilizaram os formandos do curso de Engenharia Elétrica da Universidade Federal de Rondônia (UNIR), que se organizaram e desenvolveram um protótipo de medidor de energia de baixo custo que permite o acompanhamento do consumo pelo telefone celular, sem a necessidade de conexão pela internet (SZESZ et al, 
2016). Nesse projeto, os estudantes agregaram a possibilidade do monitoramento do consumo em tempo real utilizando um amperímetro (BRAGA, 2014) conectado a uma interface bluetooth. Este texto apresenta os procedimentos adotados para desenvolvimento da hardware e do software utilizado no protótipo, bem como os testes realizados e o código fonte utilizado.

\section{Metodologia}

Para o desenvolvimento do protótipo de medidor de energia com conexão remota foram seguidas as seguintes etapas propostas por Endo, Vallim e Brito (2011):

i) Levantamento de dados;

ii) Montagem do protótipo;

iii) Elaboração de algoritmo para plataforma Android;

iv) Testes e aceitação.

\section{i) Levantamento de dados}

Foram pesquisadas as demandas para uso do instrumento em 20 residências de famílias cadastradas no serviço CadÚnico e beneficiárias da Tarifa Social de Energia Elétrica na cidade de Porto Velho/RO. Este levantamento tinha por objetivo determinar o perfil das unidades consumidoras de energia das famílias de baixa renda, para isso foram avaliados o quantitativo de consumo de eletricidade e o perfil da carga/demanda (CERON/ELETROBRAS, 2015), sendo verificado que cada residência possuía entre 2 a 5 moradores, entre zero e 65 anos.

\section{ii) Montagem do Protótipo}

Figura 1 - Desenvolvimento do Protótipo.

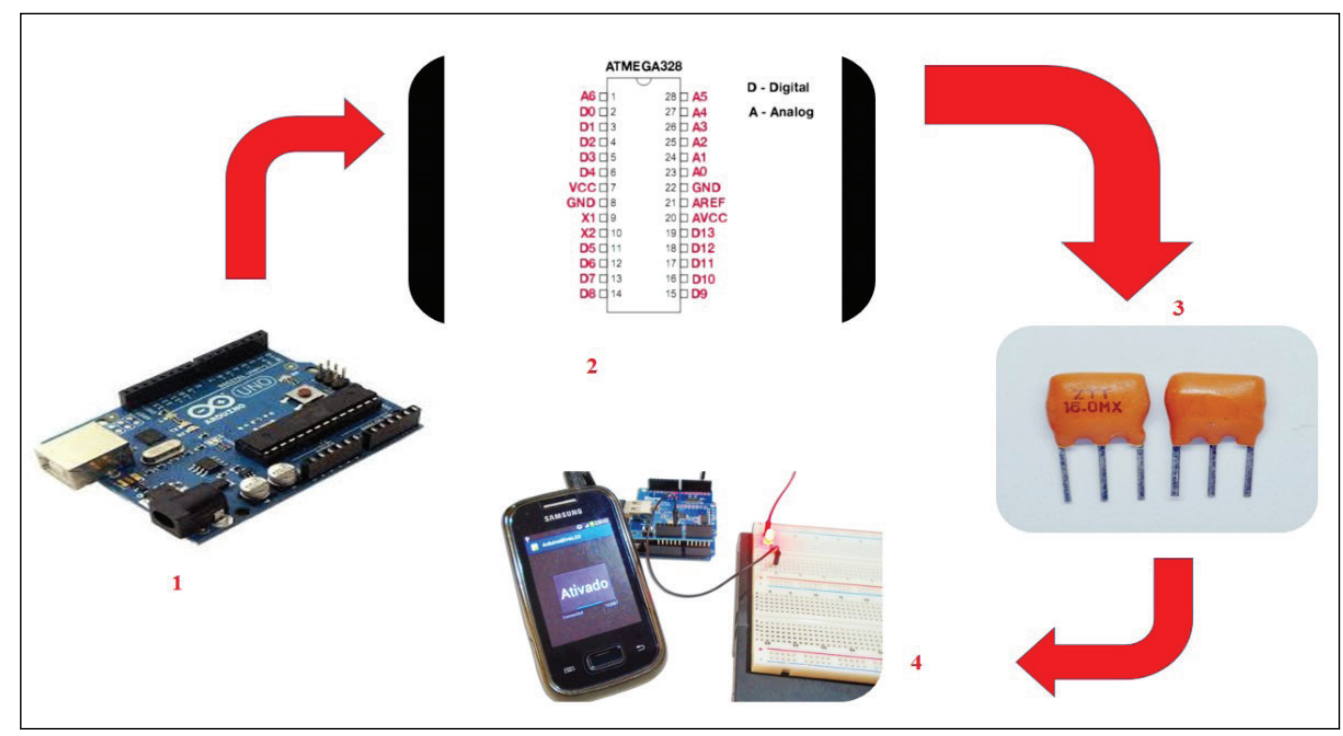

Fonte: Elaborado pelos autores (2017).

A figura 1 descreve as etapas de desenvolvimento do hardware do dispositivo de medição. Para elaboração de amperímetro por indução, foi utilizado o Arduino UNO (figura 1 - 1). O amperímetro realiza a leitura da corrente elétrica e, de maneira indireta (SADIKU, 2012), é possível correlacionar o consumo de energia kWh por meio da relação de potência de tensão (volt - V) e corrente elétrica (ampère - A) (LIMA JR., 2013). O hardware (BANZI; SHILOH, 2015) é uma placa composta por um microcontrolador baseado no ATmega 328 (figura 1 - 2), o qual possui 14 pinos digitais de entrada/saída, 6 entradas analógicas, um ressonador cerâmico $16 \mathrm{MHz}$ (figura 1 - 3) e uma conexão USB para conexão externa com placas de experimentação ou outros dispositivos (figura $1-4$ ). 
Figura 2 - Diagrama em blocos do funcionamento do amperímetro por indução

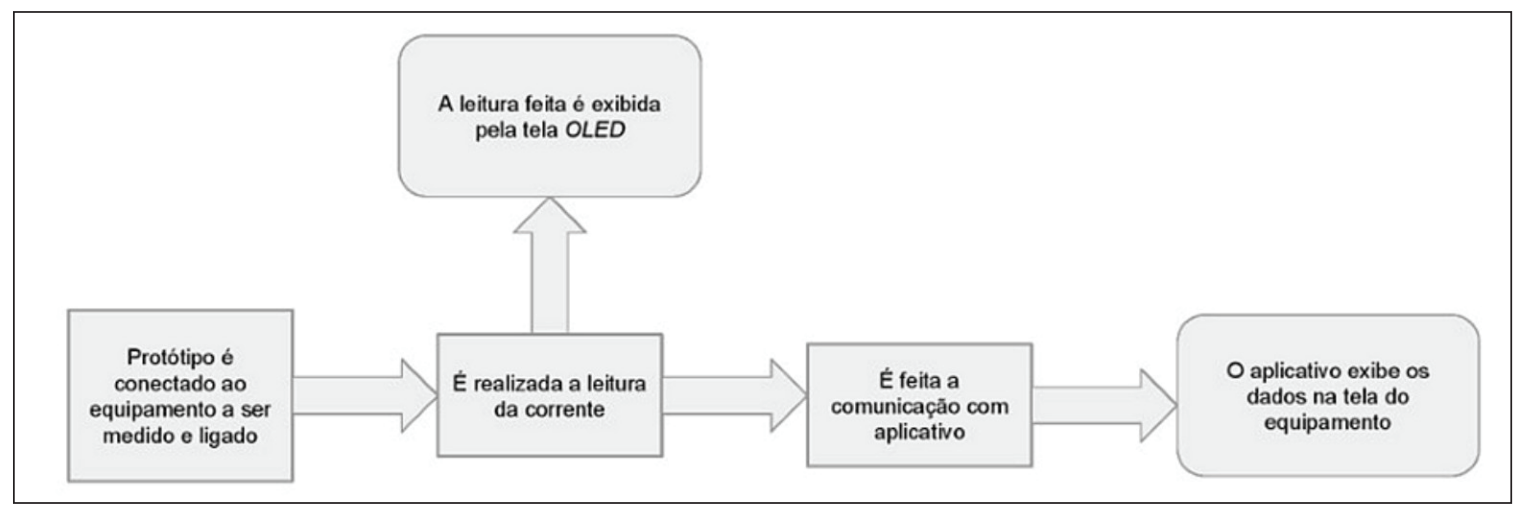

Fonte: Elaborado pelos autores (2017).

Nesse medidor de energia, a leitura do consumo deve ser realizada diretamente no equipamento, bem como remotamente em um telefone celular (CARDOS, 2014). A figura 2 apresenta o diagrama em blocos do hardware em desenvolvimento. $\mathrm{O}$ microcontrolador executa as rotinas para supervisão dos sensores e leitura da medida do consumo de energia (WIKA, 2017). Um módulo bluetooth HC-06 foi incluído para fazer a comunicação com o aplicativo remoto instalado no celular. O display Oled é um componente pequeno que exibe a leitura da medição. A tabela 1 apresenta a lista de materiais utilizados na montagem do hardware e seu respectivo custo:

Tabela 1 - Lista de materiais utilizados na montagem do protótipo

\begin{tabular}{|c|c|c|c|}
\hline Componente & Quantidade & Preço Unitário (R\$) & Preço Total (R\$) \\
\hline Tela OLED 0,96" & 1,00 & 29,90 & 29,90 \\
\hline Modulo bluetooth HC-05 & 1,00 & 32,90 & 32,90 \\
\hline Arduino UNO com microcontrolador Atmega328P & 1,00 & 49,90 & 49,90 \\
\hline Capacitor de cerâmica $22 \mathrm{pF}$ & 2,00 & 0,06 & 0,12 \\
\hline Chave tipo gangorra & 1,00 & 4,99 & 4,99 \\
\hline Pilha AA (pacote com 6) & 1,00 & 14,99 & 14,99 \\
\hline Suporte para 6 pilhas AA & 1,00 & 16,99 & 16,99 \\
\hline Sensor de corrente TA12-100 & 1,00 & 27,26 & 27,26 \\
\hline Oscilador de cristal $16 \mathrm{MHz}$ & 1,00 & 10,11 & 10,11 \\
\hline Placa fenolite $10 \times 10 \mathrm{~cm}$ & 1,00 & 3,91 & 3,91 \\
\hline Fios jumpers & 1,00 & 10,00 & 10,00 \\
\hline Estanho para solda (25mg) & 1,00 & 8,29 & 8,29 \\
\hline Percloreto de ferro $(500 \mathrm{~g})$ & 1,00 & 17,00 & 17,00 \\
\hline \multicolumn{3}{|l|}{ Total } & R\$ 226,36 \\
\hline
\end{tabular}

Fonte: Elaborado pelos autores (2017).

A figura 3, a seguir, apresenta o desenvolvimento da placa eletrônica do circuito, em que fica demonstrada a etapa de elaboração do leiaute do circuito eletrônico no emulador de desenvolvimento web CircuitLab (www.circuitlab.com). A figura $3 \mathrm{~b}$ apresenta a placa montada, com os componentes eletrônicos sendo soldados. O medidor de energia foi construído em um gabinete acrílico utilizado para acondicionar os componentes eletrônicos. Nessa montagem, todo o circuito custou $\mathrm{R} \$ 226,36$ em peças e componentes eletrônicos (tabela 1). 
Figura 3 - Confecção da placa de circuito impresso

(a) Desenvolvimento

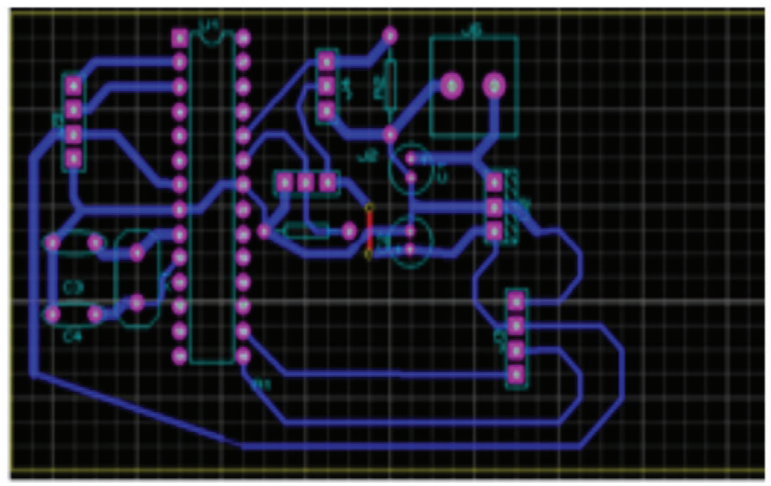

(b) Montagem

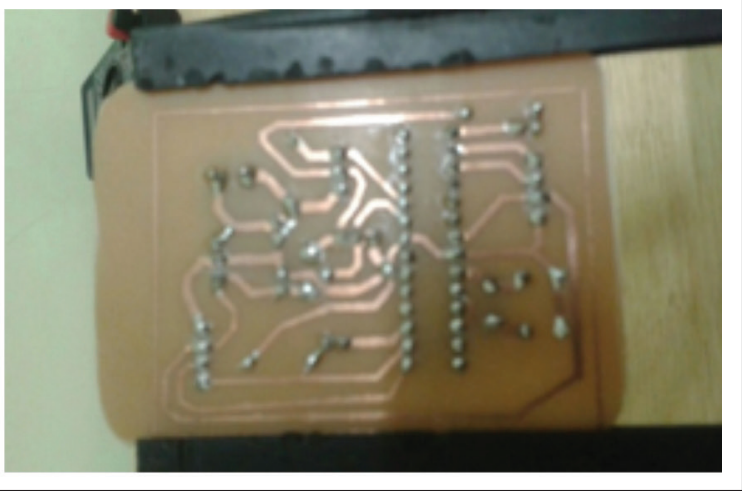

Fonte: Elaborado pelos autores (2017).

\section{iii) Elaboração do Algoritmo para plataforma Android}

O algoritmo determina as rotinas de software necessárias para funcionamento do dispositivo. Para tanto, foi desenvolvido um aplicativo no formato apk para recepção de dados. Esse software é responsável pela conexão do sistema de supervisão a um amperímetro de indução que faz a medida do consumo de energia de maneira indireta. A figura 4, a seguir, descreve resumidamente o processamento do software.

Figura 4 - Etapas do processo de comunicação remota do medidor de energia

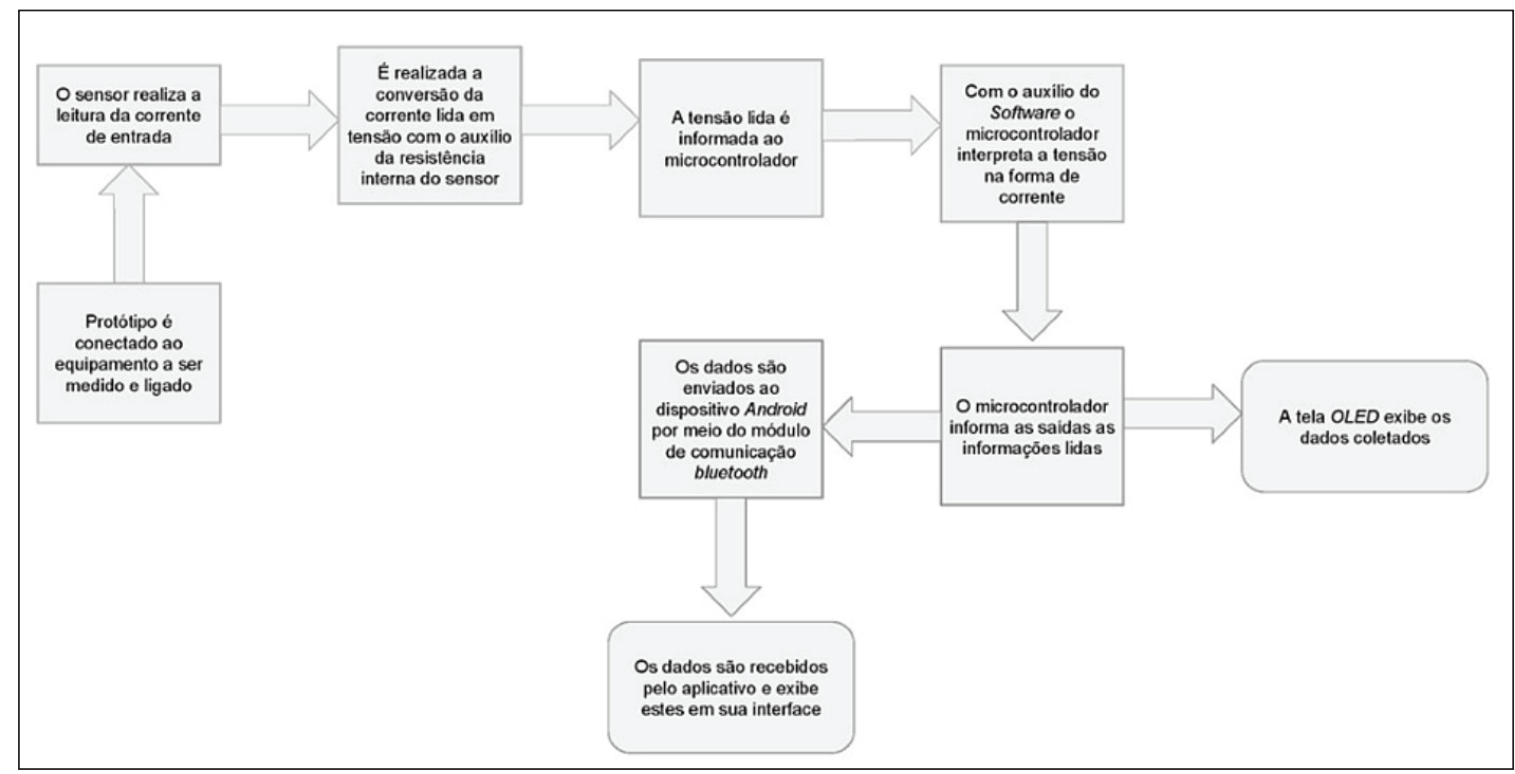

Fonte: Elaborado pelos autores (2017).

Logo após o acionamento do dispositivo, é estabelecida uma conexão com o sensor de leitura de corrente elétrica (NASCIMENTO, 2011), sendo a leitura adaptada por meio de uma conversão direta por divisor de tensão. Esse processo disponibiliza a informação para o microcontrolador, seu software interno interpreta os dados e informa para uma tela de visualização local, bem como para uma interface de dados para comunicação dluetooth. A interface disponibiliza os dados para leitura de um celular, que se conecta pela interface bluetooth (figura 5). 
Figura 5 - Desenvolvimento do programa para plataforma Android.

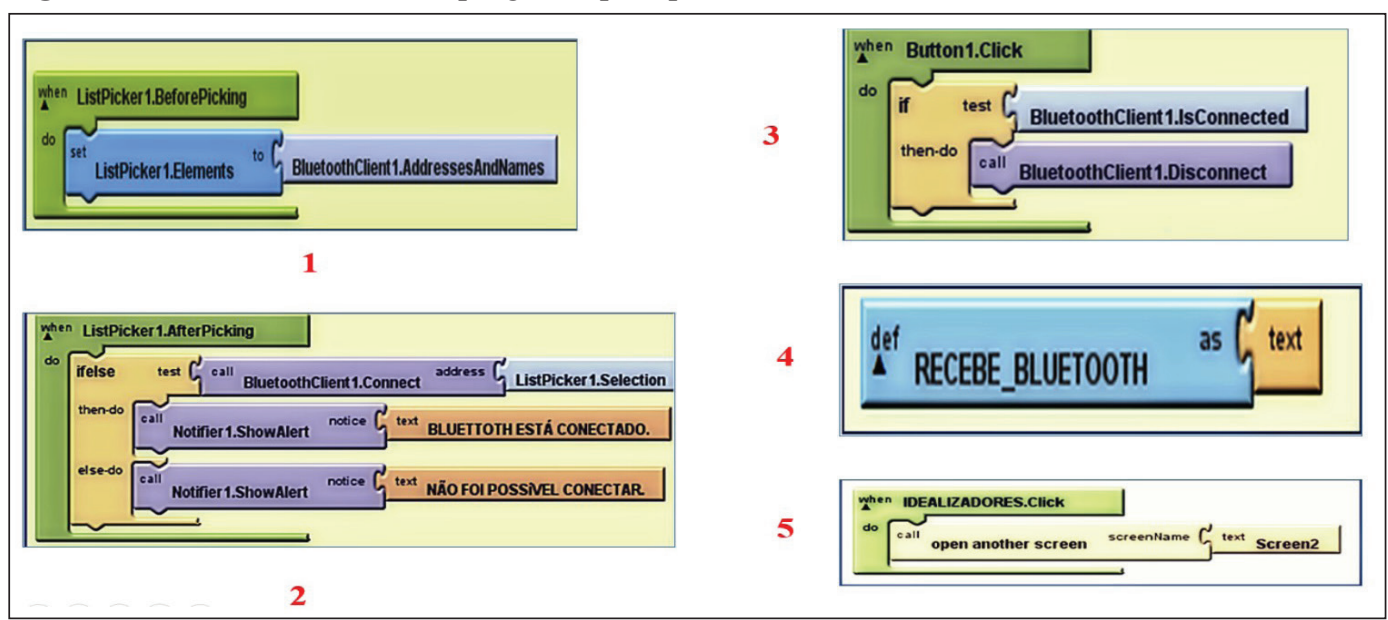

Fonte: Elaborado pelos autores (2017) e adaptado de Marinello et al (2012).

As características técnicas que permitem a comunicação bluetooth foram desenvolvidas numa plataforma de código aberto (open source) utilizando um módulo do Arduino, em que o software foi programado operação em microcontrolador ATMEGA. As leituras do equipamento são transmitidas no visor do eletrônico, bem como transmitidas para um celular com o aplicativo de leitura, este foi desenvolvido para versão Android 4.2.2 (QUEIROZ, 2016).

O desenvolvimento do aplicativo ocorreu através da versão App Inventor, disponibilizado e hospedado no site do Instituto de Tecnologia de Massachusetts - MIT. De acordo com Android Community (2017), essa versão do aplicativo oferece um aspecto de programação em blocos no sistema JAVA. Assim, o usuário necessita apenas de uma conta no Google para ter acesso (MIT, 2017). A plataforma é simples e possibilita o desenvolvimento estabelecendo suas ligações características em blocos (ABLESON et al, 2012). Cada bloco define a opção de acesso aos pontos de sinal via bluetooth, localizado pelo aparelho em uso. A programação dos blocos foi utilizada no desenvolvimento do protótipo, sendo dividida em dois blocos principais: a) acesso ao bluetooth, e b) interface homem-máquina. O processo de desenvolvimento está ilustrado na figura 6 , e seu respectivo detalhamento está na tabela 2.

Figura 6 - Tela do aplicativo de medida de energia elétrica

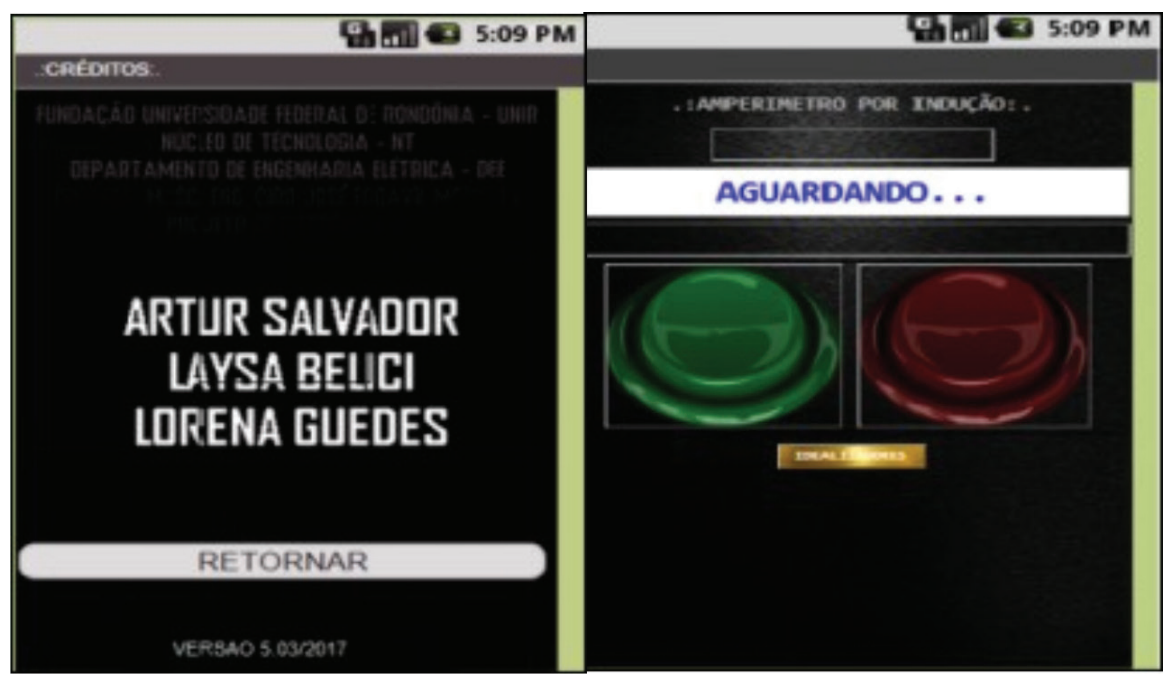

Fonte: Elaborado pelos autores (2017). 
Tabela 2 - Descritivo conforme numeração de blocos da programação do aplicativo.

\begin{tabular}{c|l}
\hline Etapa & \multicolumn{1}{c}{ Descrição } \\
\hline 1 & $\begin{array}{l}\text { Este bloco define a opção de acesso aos pontos de sinal via bluetooth localizados pelo aparelho em uso, } \\
\text { inserindo o comando para listagem de contatos recebidos pelo sinal de bluetooth. }\end{array}$ \\
\hline 2 & $\begin{array}{l}\text { Indica como positiva, ou negativa, a comunicação como escravo ou mestre entre os blocos. Bloco que alerta } \\
\text { o usuário pelo bluetooth do smartphone, indicando o recebimento dos dados enviado pelo amperímetro. }\end{array}$ \\
\hline 3 & $\begin{array}{l}\text { Bloco indicativo de conexão entre os sinais de bluetooth. Função dos botões colocados no aplicativo. No } \\
\text { caso, o botão “on”, que deve se conectar, e o botão "off”, que o desconecta. }\end{array}$ \\
\hline 4 & Mensagem de dados que deve ser recebida e lida pelo aplicativo. O espaço do texto permanece em aberto. \\
\hline 5 & $\begin{array}{l}\text { A segunda tela do aplicativo, que é a tela de créditos dos idealizadores. Há um bloco para inserir uma nova } \\
\text { tela ao aplicativo }\end{array}$ \\
\hline
\end{tabular}

Fonte: Adaptado de Fedechen, Catarino e Soler (2011).

\section{iii) Testes e aceitação}

A aferição do medidor de energia foi obtida a partir da comparação entre a medida do protótipo e um padrão de medida. O padrão utilizado foi um medidor de corrente elétrica do laboratório de eletrônica da universidade, que era era um alicate amperímetro digital Minipa ET 3200. Foram realizados ensaios com diferentes cargas resistivas, simuladas a partir de um conjunto de lâmpadas incandescentes de $60 \mathrm{~W}$ com tensões de funcionamento de $220 \mathrm{~V}$ e $127 \mathrm{~V}$. A comparação das medidas possibilitou determinar um desvio $\Delta(\%)$ que pode orientar a aceitação do dispositivo para uso nas unidades residenciais de baixa renda. Quanto menor o desvio $\Delta(\%)$, maior será a precisão do protótipo.

\section{Resultados e discussão}

Os resultados obtidos estão dispostos na tabela 3 a seguir:

Tabela 3 - Aferição comparativa do instrumento desenvolvido e o padrão de referência.

\begin{tabular}{l|c|c|c|c|c|c}
\hline \multirow{2}{*}{ Carga (W) } & \multicolumn{3}{|c|}{$\mathbf{2 2 0}$ V } & \multicolumn{2}{c}{$\mathbf{1 2 7}$ V } \\
\cline { 2 - 7 } & Padrão (A) & Protótipo (A) & $\Delta \mathbf{( \% )}$ & Padrão (A) & Protótipo (A) & $\Delta$ (\%) \\
\hline 60 & 0,26 & 0,26 & 0,00 & 0,22 & 0,22 & 0,00 \\
\hline 120 & 0,54 & 0,52 & 0,02 & 0,40 & 0,34 & 0,04 \\
\hline 180 & 0,81 & 0,81 & 0,00 & 0,58 & 0,76 & 0,01 \\
\hline 240 & 1,07 & 1,07 & 0,00 & 0,77 & 0,95 & 0,01 \\
\hline 300 & 1,34 & 1,35 & 0,74 & 0,96 & 1,16 & 0,00 \\
\hline 360 & 1,62 & 1,64 & 1,23 & 1,16 & 1,36 & 0,74 \\
\hline 480 & 1,88 & 1,92 & 2,12 & 1,35 & 1,57 & 1,29 \\
\hline 540 & 2,14 & 2,18 & 1,86 & 1,55 & 1,78 & 1,13 \\
\hline 600 & 2,44 & 2,47 & 1,23 & 1,76 & 1,97 & 1,02 \\
\hline 660 & 2,7 & 2,75 & 1,85 & 1,95 & 2,18 & 1,39 \\
\hline 720 & 2,99 & 3,04 & 1,67 & 2,15 & 2,38 & 1,27 \\
\hline 840 & 3,26 & 3,32 & 1,84 & 2,35 & 2,57 & 1,18 \\
\hline 900 & 3,53 & 3,61 & 2,26 & 2,54 & 2,78 & 1,46 \\
\hline 960 & 3,8 & 3,88 & 2,10 & 2,74 & 2,97 & 1,36 \\
\hline
\end{tabular}

Fonte: Elaborado pelos autores.

Ao iniciar o aplicativo, são exibidos os créditos e a opção para acionamento da leitura remota com dois botões digitais (figura 6). A interface com o usuário deve ser o mais simples possível, uma vez que será operada por pessoas com pouco conhecimento dos recursos digitais, tal como foi o perfil apurado no levantamento inicial. Os dados da corrente elétrica consumida são exibidos na tela do celular após o acionamento do botão verde. Nesse caso, os dados do sensor 
são transmitidos via bluetooth. Para aceitação de uso do equipamento, os critérios foram o pequeno desvio de medição apurado (tabela 3), a viabilidade de custo, bem como a praticidade de uso pelo grupo familiar em foco.

\section{Conclusão}

O medidor de energia com acesso bluetooth foi baseado em amperímetro digital por indução de baixo custo. A fabricação e a instalação do dispositivo em larga escala podem auxiliar as famílias mais carentes no controle de seu consumo de energia elétrica através de avisos indicadores de potência em seus celulares. A interface no celular é amigável e de fácil interpretação.

O protótipo desenvolvido também apresenta boa performance para medição de energia, sendo observado um desvio pequeno, que pode ser eliminado utilizando-se componentes mais precisos. Assim, os resultados obtidos foram satisfatórios, demonstrando a viabilidade e confiabilidade do dispositivo com desvio de medida menor que 5\% para diferentes cargas.

Verifica-se que o protótipo desenvolvido proporciona ao usuário facilidade no uso e praticidade, e pode ainda ser aperfeiçoado, integrando a uma plataforma de acesso pela internet, controles remotos de desligamento e acionamento, entre outros recursos, os quais podem facilitar a vida das famílias mais humildes na Amazônia.

\section{Referências}

ABLESON, W.F. et al. Android em ação: apresentando a plataforma. Rio de Janeiro: Elsevier, 2012.

AGÊNCIA NACIONAL DE ENERGIA ELÉTRICA. Relatório de tarifa social 2012: indicadores da distribuição. Disponível em: <http://www.aneel.gov.br/indicadores-da-distribuicao >. Acesso em: 06 set. 2017.

ANDROID Community. Developing in android 2017. Disponível em: $<$ https://source.android.com/source/ developing $>$. Acesso em: 16 jan. 17.

ARDUINO Uno Rev.3. Disponível em: <https://www.arduino.cc/en/Main/arduinoBoardUno>. Acesso em: 10 jan. 17.

BANZI, M.; SHILOH, M. Primeiros passos com o arduino. São Paulo: Novatec, 2015.

BRAGA, C. N. Almanaque tecnológico. São Paulo: INC, 2014.

CARDOS, L. F. C. Sistema de automação residencial via rede celular usando microcontroladores e sensores. Revista de Engenharia da Universidade Católica de Petropólis, v. 6, n. 2, p. 70-83, 2014. Disponível em <http://seer.ucp.br/ seer/index.php?journal=REVCEC\&page=article\&op=view\&path\%5B\%5D=523>Acesso em 18 jul. 17.

ELETROBRAS. Relatório de demonstrações financeiras e administração 2015. Disponível em: <http://www.diof. ro.gov.br/data/uploads/2016/04/DOE-15_04_2016.pdf>. Acesso em: 29 ago. 2016.

ENDO, W.; VALLIM, M. B. R.; BRITO, G. A. Metodologia e práticas de aprendizagem para sistemas de gerenciamento de energia: aplicações em laboratórios didáticos de graduação em engenharia. In: CONGRESSO BRASILEIRO DE EDUCAÇÃO EM ENGENHARIA, 34., 2011 Anais... Blumenau: ABENGE, 2011. Disponível em: $<$ http://198.136.59.239/ abengeorg/CobengeAnteriores/2011/sessoestec/art1829.pdf> Acesso em 20 jul. 17.

FEDECHEN, E. A.; CATARINO, I. C.; SOLER, L. Gerenciamento de mensagens internas via comunicação bluetooth dekstop-celular. Revista Científica Ciências Exatas e Tecnológicas, v.10, n.1, 2011. Disponível em <http://www. pgsskroton.com.br/seer/index.php/exatas/article/viewFile/531/501> Acesso em 20 jul 17.

LIMA JUNIOR., A.W. Eletricidade e eletrônica básica. 4 ed. Rio de Janeiro, 2013.

MARINELLO, S. H. M., et al. Determinando posições relativas de nós móveis por meio de bluetooth e aprendizados de máquina. Revista de Exatas e Tecnológicas, Rondonópolis, v. 1, n. 3, 2012. Disponível em $<$ http://retec.eti.br/retec/ index.php/retec/article/view/20> Acesso em: 20 jul. 17.

MIT. Programa app invetor. Disponível em $<$ http://appinventor.mit.edu/explore/about-us.html $>$ http://beta. appinventor.mit.edu. Acesso: 10 jan. 17.

NASCIMENTO, F. S. Controlando mão robótica com brain computer interface. COMPUTER ON THE BEACH 2011, Belém. Anais... Disponível em: <https://siaiap32.univali.br//seer/index.php/acotb/article/view/6397>. Acesso em: 20 jul. 17. 
QUEIROZ, R. S. et al. Instergas plus: insterpretação da gasometria arterial. Revista Saúde.com v. 2, n. 4, 2016. Disponível em: $<$ http://www.uesb.br/revista/rsc/ojs/index.php/rsc/article/view/473/403> Acesso em 06 set. 17.

SADIKU, M. N. O. Fundamentos de circuitos elétricos. 5 ed. Porto Alegre: AMGH, 2012.

SZESZ, A. J. et al. Tecnologias da informação e comunicação e as práticas educacionais. Revista de Engenharia e Tecnologia de Ponta Grossa, v. 8, n. 1, 2016. Disponível em: <http://www.revistaret.com.br/ojs-2.2.3/index.php/ret/ article/view/450> Acesso em: 20 jul. 17.

WIKA, A. D. Datasheet digital temperature transmitter model T12.10. Disponível em: $<$ http://de-de.wika.de/ upload/DS_TE1203_en_co_7432.pdf >.Acesso em: 10 jan. 17.

Sobre os autores

\section{Cristiano Torres do Amaral}

Engenheiro de Telecomunicações pelo Centro Universitário de Belo Horizonte - UNIBH. Mestre em Engenharia Elétrica, área de Sistemas de Computação e Telecomunicações, pela Universidade Federal de Minas Gerais - UFMG. Doutorando em Desenvolvimento Regional e Meio Ambiente na Universidade Federal de Rondônia - UNIR. Pesquisador do Grupo de Pesquisa Energia Renovável Sustentável (GPERS). Chefe da Divisão de Telecomunicações e Redes do Sistema de Proteção da Amazônia em Porto Velho - SIPAM/RO.

\section{Artur Salvador}

Engenheiro eletricista pela Universidade Federal de Rondônia - UNIR.

\section{Lorena Paula Mendonça Guedes}

Engenheira eletricista pela Universidade Federal de Rondônia - UNIR.

\section{Laya Fernanda Belici Siborde}


Engenheira eletricista pela Universidade Federal de Rondônia - UNIR.

\section{Apêndice}

Código Fonte do Hardware do Medidor de Energia com Acesso Bluetooth \#define ELECTRICITY_SENSOR A1

\#include $<$ IIC_without_ACK.h>

\#include "oledfont.c" //codetab

\#include $<$ SoftwareSerial.h $>$

\#define OLED_SDA $10 / /$ define a porta 9 com reeceptor

\#define OLED_SCL $9 / /$ define a porta 10 como tranferidor

float effective_value; //define a variavel

float amplitude_current;

SoftwareSerial mySerial $(0,1)$; // RX, TX inicia o serial

IIC_without_ACK lucky(OLED_SDA, OLED_SCL);

void $\operatorname{setup}()$

\{

lucky.Initial();

delay(10);

lucky.Fill_Screen(0xff); // limpa a tela

delay(1000);

lucky.Fill_Screen(0x00);

lucky.Char_F6x8(25,3,"Leitor de “); // printa a mensaagem

lucky.Char_F6x8(25,4,"Corrente"); // printa a mensagem

delay(2000);

lucky.Fill_Screen(0xf0);

delay(2000);

mySerial.begin(38400);//inicia o bluetooth

Serial.begin(9600); //inicia o serial

pins init(); //inicia o sensor

\}

void loop ()

\{

int sensor_max; // define a variavel

sensor_max = getMaxValue(); //dfine a variavel como o valor maximo 
//Serial.print("sensor_max = "); //printa no serial a mensagem

//Serial.println(sensor_max); //printa no serial a vaariavel

amplitude_current=(float)sensor_max/1024*5/200*1000000; //pega o valor da variavel dividi por 1024(numeero de bets) e por 5 (tensão),

//depois dividi por 200 (a resistencia do sensor) multiplicaado por 100000

que é o fator de proporcionalidade

effective_value=amplitude_current/1.414; // dividi por 1.414 para dar o valor rms (efetivo)

//Serial.println("O valor da corrente "); // printa a mensagem

Serial.println(effective value, 1 ); // printa a variavel

void pins_init()

\{

pinMode(ELECTRICITY_SENSOR, INPUT); //definee o pino do sensor como entrada

int getMaxValue()

\{

float sensorValue; //define a variaveel

int sensorMax $=0$;

uint32_t start time $=$ millis () ;

while $(($ millis()-start_time $)<1000) / /$ faz uma leitura a cada segundo

\{

sensorValue $=$ analogRead(ELECTRICITY_SENSOR);

if (sensorValue $>$ sensorMax)

\{

//compara os valores de sensorMax e sensor Value e armazena o maior valor sensorMax = sensorValue;

\}

\}

return sensorMax; //inicia a programação

mySerial.println(sensorValue); //printa o maior valor analisado

lucky.Fill_Screen(0x00);

lucky.Char_F6x8(15,2,"Corrente efetiva: “);

// printnum(36,4,effective_value);

lucky.Char_F6x8(60,5,"A");

delay(3000);

\} 